\title{
Valoración prospectiva del capital intelectual de la Universidad de Atacama, mediante la técnica Delphi
}

\author{
Prospective evaluation of intellectual capital of Universidad de Atacama, \\ through the Delphi technique
}

\author{
José Luis Silva Munar $^{1} \quad$ Planck Barahona Urbina $^{1} \quad$ Carlos Galleguillos Cortés $^{1}$
}

Recibido 12 de junio de 2013, aceptado 6 de mayo de 2014

Received: June 12, 2013 Accepted: May 6, 2014

\begin{abstract}
RESUMEN
El objetivo de este trabajo ha sido medir las variables de capital intelectual en la Universidad de Atacama, con una mirada prospectiva para los próximos diez años. Para ello se ha utilizado la metodología Delphi. Esta técnica se ha complementado con una herramienta matemática denominada Aproximación de Perfiles Cualitativos, basada en una serie de variables predeterminadas. Este trabajo describe el concepto de capital intelectual dividido en tres variables, esto es; el capital humano, el capital estructural y el capital relacional, pues esta clasificación admite en primer lugar elementos como el valor del conocimiento creado por las personas. En segundo lugar el conocimiento que se ha hecho explícito y documentado y, finalmente, el intercambio de información con externos. Para llevar a cabo el estudio se ha realizado una encuesta, cuyo fin ha sido considerar una serie de variables relacionadas con la percepción. Los resultados han puesto de manifiesto que la percepción de la comunidad no ha sido muy favorable. Sin embargo, las opiniones han servido para generar un acercamiento en la medición de los intangibles y la gestión del conocimiento.
\end{abstract}

Palabras clave: Gestión del conocimiento, capital intelectual, metodología Delphi, dirección estratégica, valoración prospectiva.

\begin{abstract}
The aim of this study has been to measure the variables of Intellectual Capital at Universidad de Atacama, with a prospective look for the next ten years. To do this, we have used the Delphi methodology. This technique has been supplemented with a mathematical tool called Qualitative Profiling Approach, based on a series of predetermined variables. This work describes the concept of Intellectual Capital divided into three variables, namely: Human Capital, Structural Capital and Relational Capital, since this classification supports firstly elements like the value of knowledge created by the people, secondly knowledge that has been expressed explicitly and documented, finally by external information exchange. To carry out the study a survey was conducted, whose purpose has been to consider a number of variables related to perception. The results have shown that the perception of the community has not been very favorable. However, the opinions have been useful to generate approaches in measuring intangibles and Knowledge Management.
\end{abstract}

Keywords: Knowledge management, intellectual capital, Delphi methodology, strategic management, prospective evaluation.

\footnotetext{
1 Departamento de Industria y Negocios. Facultad de Ingeniería. Universidad de Atacama. Copiapó, Chile. E-mail: jose.silva@industria.uda.cl; planck.barahona@uda.cl; carlos.galleguillos@uda.cl
} 


\section{INTRODUCCIÓN}

El objetivo primordial de toda organización es la búsqueda del desempeño superior, representado en las instituciones públicas por su énfasis en el bienestar social y por la maximización de la riqueza en las instituciones privadas.

En la antigüedad, las organizaciones verificaban su éxito mediante indicadores financieros, cuyos resultados eran el reflejo de lo que estaba ocurriendo con las inversiones y el valor económico añadido.

En la medida que el entorno fue evolucionando, como consecuencia de las transformaciones políticas, económicas, sociales y tecnológicas, las organizaciones comenzaron a interactuar en un ambiente global, dinámico y competitivo, donde lo único constante es el cambio. En este sentido, los activos intangibles cobran relevancia porque articulan, fortalecen y estimulan los procesos internos, la orientación al cliente y, como consecuencia, un resultado financiero óptimo, que dependiendo de las capacidades y recursos de las organizaciones permiten alcanzar la ventaja competitiva.

De este modo se consigna que la visión y la estrategia de la organización son las herramientas fundamentales para encaminar los esfuerzos individuales y colectivos. Dentro de ella, los elementos intangibles deben estar alineados con el objetivo de crear valor desde la perspectiva de facilitar la toma de decisiones estratégicas y de mejorar la percepción de la sociedad en general.

De acuerdo con la opinión de muchos teóricos, las universidades públicas deben estar preparadas para este acelerado cambio. Un aporte importante en esta materia podría ser generar proyectos que apunten a nuevos desafíos. Creemos que pronosticar el futuro y, en este caso, medir el capital intelectual ayudaría a tomar decisiones hoy.

\section{BREVE REVISIÓN DE LA LITERATURA}

En 1990 ya se hablaba de la capacidad de hacer rendir más los activos de la organización y que estos estaban reflejados en la capacidad de dirección, para crear ventajas competitivas. En la actualidad, este incipiente concepto de capacidad ha sido definido como un recurso intangible [1].
La mayoría de los teóricos reconocen que Sveiby [2] fue quien desarrolló la primera teoría de la organización del conocimiento, donde apoyaba la idea de que el valor total de la organización está formado por el patrimonio tangible y los elementos intangibles, fundamentado en la habilidad de las personas para actuar en situaciones diversas, su educación, la experiencia, los valores y las habilidades sociales.

La definición de capital intelectual presentada por Joia [3] se refiere a crear, conseguir y gestionar eficazmente todos aquellos elementos intelectuales necesarios para conseguir los objetivos de la organización y llevar a término con éxito sus estrategias. Si bien este concepto se ha justificado por la diferencia que existe entre el valor contable de la acción y su valor bursátil de sociedades anónimas abiertas, ha sido utilizada por distintos tipos de organizaciones, pues todas ellas son percibidas por una comunidad y todas ellas, deben crear ventajas competitivas.

El capital intelectual entonces son todos aquellos elementos no tangibles que en un marco estratégico específico conducen a la creación de valor de los tradicionales activos tangibles [4-5].

Mientras que en la era industrial las organizaciones eran construidas con una considerable dependencia de los recursos naturales, grandes terrenos, activos físicos, mano de obra y capital financiero, en la nueva economía del conocimiento y la información la fuente de riqueza primordial son el empleo de tal conocimiento y el uso imaginativo de la tecnología [6-7].

Actualmente, algunas organizaciones con sus métodos tradicionales de gestión basados en aspectos contables y financieros han requerido de información complementaria para la toma de decisiones. Así, han concentrado su atención en los valores intangibles, pues han entendido que ayudan a generar ventajas para el futuro [8].

Para Didou [9], el avance en la tecnología de la información y la comunicación, y su amplia introducción en las diferentes actividades, ha generado una dinámica sin precedentes, que involucra a la organización con su entorno materializado en sus relaciones con sus inversionistas, clientes, 
proveedores, gobierno, entre otros. Lo anterior presiona a todo tipo de organizaciones, incluyendo a los sistemas de educación a aumentar su capacidad de subsistencia en este medio de incertidumbre, para transformarse y resistir el cambio.

Es innegable que las instituciones de educación juegan un rol importante en las sociedades. De hecho, un factor clave para generar capacidades competitivas de los países [10], en su crecimiento económico y en su desarrollo social, ha sido el mejoramiento de los sistemas formales de educación. Por lo tanto, para competir en este escenario es preciso que la Universidad como ente formativo de la intelectualidad transforme sus utopías y sus modelos de gestión.

Las instituciones de enseñanza superior [11] tendrán que mostrarse más flexibles para adaptarse a las necesidades de la sociedad y preverlas. Esto se aplica sobre todo a los países en desarrollo, en los que es urgente establecer nuevos modelos universitarios para propiciar sinergias disciplinarias y geográficas, así como cooperación en el plano regional e internacional.

Cuando Rodríguez [12] cita a Rainey y Bozeman destaca que los autores defendían la idea de que las diferencias entre firmas privadas e instituciones públicas están dadas por la complejidad y ambigüedad de las metas, la estructura organizativa, el grado de formalización, las actitudes y valores relativos al trabajo. La gestión de las universidades no queda exenta a este concepto, principalmente en las sociedades occidentales [13], pues esta problemática se evidencia cuando se intentan abordar cuestiones relacionadas con los procesos de evaluación institucional, acreditación de carreras, diseño de presupuestos, presentación de proyectos con fines específicos, creación de nuevas carreras, entre otras.

Creemos que es un desafío entonces generar estudios de esta índole en instituciones de educación superior pública. Y más aún, generar estudios prospectivos donde se analicen escenarios futuros que podrían ayudar a tomar decisiones en la gestión estratégica. Los ejercicios prospectivos dan la posibilidad de visualizar las tendencias dominantes. Son una herramienta que ayuda a visualizar las brechas entre el futuro deseado y el futuro previsible.
Visualización en donde se genera una capacidad de realizar seguimientos, para que por medio de evidencias observables se pueda hacer la gestión correctiva si fuese necesaria [14].

En esencia, visualizar el futuro nace como una necesidad cuando este futuro no puede ser visto como una simple prolongación del pasado. Tal visualización consiste en tener un panorama de los futuros posibles, como son los presentados en este proyecto mediante las variables de capital intelectual, representados cada uno de ellos en un escenario determinado.

La técnica Delphi [15] es pionera en el desarrollo de estudios de prospectiva. Esta técnica tiene por objeto la construcción de escenarios, con relación al comportamiento de una o varias variables tomadas en consideración. Carreño [16] aduce que el método Delphi ha sido utilizado frecuentemente como sistema para obtener información sobre el futuro. Es definido como un método de estructuración de un proceso de comunicación grupal, que permite a individuos, como un todo, tratar un problema complejo. De acuerdo con lo expuesto, los intangibles son un tema complejo y difícil de medir. Si esta técnica la unimos con la metodología denominada Aproximación por Perfiles Cualitativos [15], podríamos llegar a un refinamiento de los datos en cuestión. Esta metodología deduce matemáticamente un valor síntesis, por el concepto topológico de la función distancia o definición de una medición en un conjunto dado.

La metodología trabaja con la mejor aproximación al perfil de opinión del grupo. Para ello se recurre al concepto de distancia entre n-uplas. Aquel perfil básico de rango que mejor se aproxima, y por lo tanto su cualificación asociada o a la cual representa, será la cualificación que mejor representa, como síntesis, la opinión de ese grupo. Y es eso lo que se busca, sobre todo en las materias que en una universidad pública son un desafío medir.

\section{INDICADORES PARA LA MEDICIÓN DE CAPITAL INTELECTUAL}

Para el siguiente estudio ocuparemos la definición de capital intelectual que nos ofrece Molina, Arango y Botero [10], que hacen referencia a un conjunto de recursos intangibles de carácter 
estratégico que, a pesar de contribuir a la creación de valor organizativo, no figuran en los estados financieros de la organización. De acuerdo con este concepto, capital intelectual se divide en las siguientes variables: capital humano como el conjunto de conocimientos, habilidades, formación, aptitudes, motivación, destrezas y experiencia de los empleados de la organización, que posibilitan tener los recursos y las capacidades adecuadas para el futuro. Capital estructural como el que representa el conocimiento que ha pasado de estar localizado en las personas y sus relaciones, a incorporarse en las estructuras organizativas, como es el caso de la cultura organizativa, las rutinas, políticas o procedimientos organizativos, y capital relacional como el valor de las relaciones de la organización con terceros, centrado principalmente en las relaciones con los clientes, los proveedores, los accionistas y las administraciones, adoptando así una perspectiva externa.

Esta estructura de los componentes del capital intelectual será la base fundamental en la medición de los intangibles aplicado a la Universidad de Atacama, pues de esta figura saldrán los indicadores con los cuales se consultará las opiniones para los próximos diez años. Por lo señalado por Sleimen [17], si se observará a la universidad pública en términos de capital humano, capital estructural y capital relacional se podría considerar que las competencias de los docentes e investigadores son un elemento crucial en la variable de capital humano. Por otra parte, la producción científica como publicaciones, patentes y desarrollos serían elementos cruciales de la variable capital estructural. Finalmente, la cooperación y las alianzas con otras universidades, centros de formación o instituciones de la comunidad serían parte protagónica de su capital relacional.

\section{METODOLOGÍA}

De acuerdo con la metodología Delphi se confeccionó un cuestionario basado en variables del capital intelectual perteneciente a un estudio de la Universidad Jaume I De Castellón, de España [18]. Las variables que se usaron para la medición del capital intelectual han sido las que se han mencionado anteriormente. Estas variables a su vez han sido subdivididas en dimensiones, y estas dimensiones a su vez en ítems.
La investigación ha sido desarrollada utilizando la técnica Delphi con el objeto de medir el capital intelectual en la Universidad de Atacama considerando la percepción para los próximos diez años. La selección del panel de expertos se desarrolló con una base de datos de personas que conocen o tienen cierto conocimiento del concepto de capital intelectual aplicado a una universidad pública. $\mathrm{La}$ encuesta se desarrolló con dos rondas de consultas, ambas en forma anónima. El uso de este criterio en un ejercicio Delphi define que la razón del proceso de retroalimentación, con la recolección de opiniones de los participantes, no es otra cosa que obtener la estabilización de tales opiniones, es decir, una retroalimentación controlada [15].

En este caso el panel fue compuesto por directivos, académicos y profesionales de la Universidad de Atacama y de otras universidades, alumnos regulares y titulados de nuestra casa de estudio, autoridades públicas y privadas, gerentes de áreas de empresas, gerentes de recursos humanos. Se invitó a participar en la primera ronda Delphi a sesenta y tres personas. El número total de participantes que concretó la encuesta ha sido de cincuenta personas. En la segunda circulación respondieron cuarenta y cuatro de ellos, número definitivo y suficiente para iniciar la evaluación final.

El número es aceptable, por la calidad de sus opiniones, porque está entre la horquilla recomendada de diez y treinta, pues exitosos Delphi se han llevado a cabo antes con veinte expertos iniciales [19]. La opinión de los participantes fue expresada en una escala conformada por los numerales del uno al siete, numerales cuyo significado viene expresado en la Tabla 1 por las siguientes expresiones linguiísticas:

Tabla 1. Escala Likert con expresiones lingüísticas para la opinión de medición.

\begin{tabular}{|c|l|}
\hline Numeral & \multicolumn{1}{|c|}{ Expresión lingüística asociada } \\
\hline 1 & Severo desmejoramiento \\
\hline 2 & Desmejoramiento \\
\hline 3 & Leve desmejoramiento \\
\hline 4 & Se mantendrá en los mismos niveles \\
\hline 5 & Leve mejoramiento \\
\hline 6 & Mejoramiento \\
\hline 7 & Severo mejoramiento \\
\hline
\end{tabular}

Debido a que la opinión de los participantes presenta una marcada variabilidad de opinión, se aplicó 
la técnica denominada Aproximación de Perfiles Cualitativos, es decir, se tabuló con aquel perfil básico de rango que mejor representa, como síntesis, la opinión de esos participantes. Los cuestionarios han sido respondidos por 44 personas.

Entonces, según la metodología aludida, se determina que el cálculo de la distancia entre el perfil de opinión del grupo y los 7 perfiles básicos de rangos es: $i=1$ a 7. Esta propuesta de Arapé [15] simboliza la distancia entre el perfil de opinión del grupo y cualquiera de los perfiles básicos de rango de la forma (1):

$$
d(\overline{O G}, \overline{C i})
$$

Donde:

$\overline{O G}=$ Escala de medición que asocia números a cada categoría, es decir, el perfil de opinión del grupo. $\overline{\mathrm{Ci}}=$ Los 7 perfiles básicos de rangos, en este caso $\mathrm{i}=1$ a 7

Basados en los conceptos de topología en Matemática, podemos decir que en este caso existen hasta 44 funciones distancias, pues hay 44 opiniones consultadas, representadas en la forma (2), que en definitiva es la función distancia de la técnica de aproximación de perfiles cualitativos.

$$
\|\|_{i}=\left[\sum_{j=1}^{44}\left|\overline{O G j}-\overline{C i_{j}}\right|^{i}\right]^{1 / i}
$$

Donde:

\section{$\overline{C i}_{j}^{l}=$ Componente j-ésimo de $\overline{C i}$}

$\overline{O G} j=$ Componente j-ésimo de la 44-upla $\overline{O G}$

Lo usual es comenzar a trabajar con la distancia NORMA-1- ( ||$\left.\cdot \mid \|_{1}\right)$. Si resulta que hay dos o más perfiles básicos de rangos con igual distancia al perfil de opinión del grupo; entonces se recurre a usar la distancia NORMA-2- $\left(\| .\left.\right|_{2}\right)$, y así sucesivamente, hasta romper la condición de igualdad entre distancias de perfiles básicos de rango y el perfil de opinión del grupo.

\section{RESULTADOS}

Los resultados obtenidos se basaron en las variables y dimensiones que se presentan en las Tablas 2, 3 y 4.
Tabla 2. Dimensiones y subdimensiones de capital

\begin{tabular}{|c|c|}
\hline \multicolumn{2}{|c|}{ Comportamiento del indicador } \\
\hline Dimensiones & Indicador \\
\hline \multirow{3}{*}{$\begin{array}{l}\text { Conocimientos } \\
\text { técnicos }\end{array}$} & $\begin{array}{l}\text { Cualificación técnica del personal aca- } \\
\text { démico }\end{array}$ \\
\hline & Cualificación técnica de los directivos \\
\hline & $\begin{array}{l}\text { Cualificación técnica del personal ad- } \\
\text { ministrativo }\end{array}$ \\
\hline \multirow{3}{*}{ Experiencia } & Experiencia de la dirección. \\
\hline & $\begin{array}{l}\text { Experiencia en otros ámbitos empresa- } \\
\text { riales de los actuales }\end{array}$ \\
\hline & $\begin{array}{l}\text { Experiencia profesional de los emplea- } \\
\text { dos. }\end{array}$ \\
\hline \multirow{4}{*}{$\begin{array}{l}\text { Habilidades de } \\
\text { liderazgo }\end{array}$} & Capacidad de comunicación. \\
\hline & $\begin{array}{l}\text { Fidelidad a la universidad y a los pro- } \\
\text { pios compromisos. }\end{array}$ \\
\hline & $\begin{array}{l}\text { Ánimo para actuar, fundado en la con- } \\
\text { fianza del éxito en situaciones difíciles. }\end{array}$ \\
\hline & $\begin{array}{l}\text { Capacidad de regular el comportamien- } \\
\text { to y las relaciones humanas por un con- } \\
\text { junto de reglas morales. }\end{array}$ \\
\hline \multirow{3}{*}{$\begin{array}{l}\text { Habilidad de } \\
\text { trabajo en } \\
\text { equipo }\end{array}$} & $\begin{array}{l}\text { Capacidad de integrar y movilizar a } \\
\text { miembros de un equipo. }\end{array}$ \\
\hline & $\begin{array}{l}\text { Habilidad en el manejo de conflictos } \\
\text { mediante la autocrítica y la apertura a } \\
\text { la mejora continua, y no mediante el } \\
\text { recurso de la legalidad. }\end{array}$ \\
\hline & $\begin{array}{l}\text { Capacidad de diálogo y de aceptación } \\
\text { de opiniones diversas. }\end{array}$ \\
\hline \multirow{4}{*}{$\begin{array}{l}\text { Habilidad } \\
\text { directiva para } \\
\text { la prospectiva } \\
\text { y el anticipo de } \\
\text { retos }\end{array}$} & $\begin{array}{l}\text { Capacidad directiva de anticipación a } \\
\text { nuevas oportunidades y amenazas }\end{array}$ \\
\hline & $\begin{array}{l}\text { Orientación emprendedora de la direc- } \\
\text { ción. }\end{array}$ \\
\hline & $\begin{array}{l}\text { Habilidad directiva para la gestión del } \\
\text { cambio. }\end{array}$ \\
\hline & $\begin{array}{l}\text { Conducta en situaciones de crisis basa- } \\
\text { da en el aprovechamiento activo para } \\
\text { descubrir nuevas oportunidades y no en } \\
\text { el esperar y ver. }\end{array}$ \\
\hline \multirow[t]{3}{*}{$\begin{array}{l}\text { Rotación de } \\
\text { tareas }\end{array}$} & $\begin{array}{l}\text { Grado de implantación de sistemas } \\
\text { como variedad del trabajo, autonomía } \\
\text { en la preparación y ejecución del traba- } \\
\text { jo, información sobre los resultados del } \\
\text { trabajo, ampliación de tareas o rotación } \\
\text { de puestos. }\end{array}$ \\
\hline & Polivalencia en la definición de tareas. \\
\hline & Rotación del trabajo \\
\hline \multirow[t]{3}{*}{ Formación } & $\begin{array}{l}\text { Eficacia en el desarrollo de programas } \\
\text { de formación adecuados, para que la } \\
\text { base de conocimientos tecnológicos } \\
\text { de la organización permita su comuni- } \\
\text { cación con organizaciones de difusión } \\
\text { de innovaciones y de transferencia de } \\
\text { tecnología. }\end{array}$ \\
\hline & $\begin{array}{l}\text { Esfuerzo de la institución para la for- } \\
\text { mación y el desarrollo del personal. }\end{array}$ \\
\hline & $\begin{array}{l}\text { Eficacia de la estrategia de formación en } \\
\text { términos de desarrollo de las competen- } \\
\text { cias clave para la organización. }\end{array}$ \\
\hline
\end{tabular}
intelectual humano. 


\begin{tabular}{|l|l|}
\hline $\begin{array}{l}\text { Proyectos } \\
\text { innovadores } \\
\text { implantados }\end{array}$ & $\begin{array}{l}\text { Formación e inversión de equipos de } \\
\text { I+D integrados por miembros de diver- } \\
\text { sas áreas funcionales. }\end{array}$ \\
\hline
\end{tabular}

Tabla 3. Dimensiones y subdimensiones de capital intelectual estructural.

\begin{tabular}{|c|c|}
\hline \multirow{3}{*}{$\begin{array}{l}\text { Conocimientos } \\
\text { relativos a cuestiones } \\
\text { externas protegidas o } \\
\text { no legalmente }\end{array}$} & $\begin{array}{l}\text { Capacidad de la institución para } \\
\text { crear y proteger patentes de pro- } \\
\text { ducto. }\end{array}$ \\
\hline & $\begin{array}{l}\text { Capacidad de la institución para } \\
\text { crear y proteger patentes de pro- } \\
\text { ceso. }\end{array}$ \\
\hline & $\begin{array}{l}\text { Rendimiento de las patentes en } \\
\text { procesos propios y externos a la } \\
\text { organización. }\end{array}$ \\
\hline \multirow{3}{*}{$\begin{array}{l}\text { Conocimientos } \\
\text { relativos a cuestiones } \\
\text { internas protegidas o } \\
\text { no legalmente }\end{array}$} & $\begin{array}{l}\text { Grado de conocimientos del mer- } \\
\text { cado, de las marcas y los símbo- } \\
\text { los de imagen de la organización } \\
\text { y/o sus productos. }\end{array}$ \\
\hline & $\begin{array}{l}\text { Reputación de institución líder en } \\
\text { el mercado. }\end{array}$ \\
\hline & $\begin{array}{l}\text { Imagen pública de la organiza- } \\
\text { ción en su entorno (reputación de } \\
\text { institución innovadora y creativa, } \\
\text { de calidad, de tecnología avanza- } \\
\text { da). }\end{array}$ \\
\hline \multirow{3}{*}{$\begin{array}{l}\text { Disponibilidad } \\
\text { de mecanismos } \\
\text { o programas de } \\
\text { mejora eficaces en la } \\
\text { organización. }\end{array}$} & $\begin{array}{l}\text { Implantación de sistemas de ase- } \\
\text { guramiento de la calidad. }\end{array}$ \\
\hline & $\begin{array}{l}\text { Implantación de sistemas de ca- } \\
\text { lidad total. }\end{array}$ \\
\hline & $\begin{array}{l}\text { Implantación de sistemas de ges- } \\
\text { tión del conocimiento. }\end{array}$ \\
\hline \multirow{4}{*}{$\begin{array}{l}\text { Disponibilidad de } \\
\text { mecanismos eficaces } \\
\text { de transmisión y } \\
\text { comunicación de } \\
\text { conocimiento }\end{array}$} & $\begin{array}{l}\text { Disponibilidad de sistemas for- } \\
\text { males de transmisión y comuni- } \\
\text { cación de conocimientos (como } \\
\text { informes escritos o reuniones } \\
\text { programadas). }\end{array}$ \\
\hline & $\begin{array}{l}\text { Disponibilidad de sistemas infor- } \\
\text { males de transmisión y comuni- } \\
\text { cación de conocimiento. }\end{array}$ \\
\hline & $\begin{array}{l}\text { Disponibilidad de procedimien- } \\
\text { tos de control y coordinación } \\
\text { descentralizados e informales } \\
\text { que estimulan la participación de } \\
\text { los empleados (como círculos de } \\
\text { calidad). }\end{array}$ \\
\hline & $\begin{array}{l}\text { Compartición de conocimiento } \\
\text { mediante la polivalencia en la } \\
\text { definición de tareas y puestos de } \\
\text { trabajo. }\end{array}$ \\
\hline \multirow{4}{*}{$\begin{array}{l}\text { Conocimiento de } \\
\text { las competencias } \\
\text { distintivas origen } \\
\text { de las ventajas } \\
\text { competitivas de la } \\
\text { organización }\end{array}$} & $\begin{array}{l}\text { Conocimiento por la institución } \\
\text { de sus fortalezas y debilidades en } \\
\text { marketing. }\end{array}$ \\
\hline & $\begin{array}{l}\text { Conocimiento por la institución } \\
\text { de sus fortalezas y debilidades en } \\
\text { producción. }\end{array}$ \\
\hline & $\begin{array}{l}\text { Conocimiento por la institución } \\
\text { de sus fortalezas y debilidades en } \\
\text { tecnología e I+D. }\end{array}$ \\
\hline & $\begin{array}{l}\text { Conocimiento por la institución } \\
\text { de sus fortalezas y debilidades } \\
\text { financieras. }\end{array}$ \\
\hline
\end{tabular}

\begin{tabular}{|c|c|}
\hline $\begin{array}{l}\text { Localización } \\
\text { geográfica de la } \\
\text { organización }\end{array}$ & $\begin{array}{l}\text { Recursos de conocimiento com- } \\
\text { partido merced a la localización } \\
\text { de la organización en distritos de } \\
\text { la misma industria. }\end{array}$ \\
\hline \multirow{4}{*}{$\begin{array}{l}\text { Alianzas estratégicas } \\
\text { con otras } \\
\text { organizaciones }\end{array}$} & $\begin{array}{l}\text { Intensidad y estructuración de las } \\
\text { alianzas estratégicas establecidas } \\
\text { por la institución con competido- } \\
\text { res para crear conocimiento. }\end{array}$ \\
\hline & $\begin{array}{l}\text { Intensidad y estructuración de las } \\
\text { alianzas estratégicas establecidas } \\
\text { por la institución con clientes } \\
\text { para crear conocimiento. }\end{array}$ \\
\hline & $\begin{array}{l}\text { Intensidad y estructuración de las } \\
\text { alianzas estratégicas establecidas } \\
\text { por la institución con centros tec- } \\
\text { nológicos, universidades u otras } \\
\text { organizaciones para crear cono- } \\
\text { cimiento. }\end{array}$ \\
\hline & $\begin{array}{l}\text { Intensidad y estructuración de las } \\
\text { alianzas estratégicas establecidas } \\
\text { por la institución con proveedo- } \\
\text { res para crear conocimiento. }\end{array}$ \\
\hline \multirow{3}{*}{ Inversión en I + D } & $\begin{array}{l}\text { Compromiso de recursos de la } \\
\text { institución en actividades de } \\
\text { I+D+I. }\end{array}$ \\
\hline & $\begin{array}{l}\text { Compromiso de recursos de la } \\
\text { institución en implantación de las } \\
\text { tecnologías de la información. }\end{array}$ \\
\hline & $\begin{array}{l}\text { Vinculación de los sistemas de } \\
\text { promoción y recompensa a la in- } \\
\text { novación. }\end{array}$ \\
\hline
\end{tabular}

Tabla 4. Dimensiones y subdimensiones de capital intelectual relacional.

\begin{tabular}{|c|c|}
\hline \multirow{2}{*}{$\begin{array}{l}\text { Conocimiento } \\
\text { de los clientes } \\
\text { relevantes }\end{array}$} & $\begin{array}{l}\text { Disponibilidad de sistemas y re- } \\
\text { cursos para el estudio continuo y } \\
\text { actualizado de los alumnos. }\end{array}$ \\
\hline & $\begin{array}{l}\text { Capacidad para obtener informa- } \\
\text { ción de los alumnos y tendencias } \\
\text { actuales del mercado. }\end{array}$ \\
\hline \multirow{3}{*}{$\begin{array}{l}\text { Conocimiento de } \\
\text { las variables clave } \\
\text { para satisfacer a los } \\
\text { clientes }\end{array}$} & $\begin{array}{l}\text { Compromiso de la institución con } \\
\text { la satisfacción de los alumnos. }\end{array}$ \\
\hline & $\begin{array}{l}\text { Disponibilidad de sistemas y re- } \\
\text { cursos para el estudio de las ne- } \\
\text { cesidades y expectativas de los } \\
\text { alumnos. }\end{array}$ \\
\hline & $\begin{array}{l}\text { Disponibilidad de sistemas y re- } \\
\text { cursos para el estudio de la satis- } \\
\text { facción de los alumnos. }\end{array}$ \\
\hline \multirow{2}{*}{$\begin{array}{l}\text { Mecanismos para } \\
\text { captar información } \\
\text { sobre los } \\
\text { competidores }\end{array}$} & $\begin{array}{l}\text { Capacidad para obtener informa- } \\
\text { ción de los competidores. }\end{array}$ \\
\hline & $\begin{array}{l}\text { Disponibilidad de sistemas y re- } \\
\text { cursos para el análisis de la com- } \\
\text { petencia. }\end{array}$ \\
\hline \multirow{2}{*}{$\begin{array}{l}\text { Relación con el } \\
\text { cliente para crear } \\
\text { conocimiento }\end{array}$} & $\begin{array}{l}\text { Importancia de la cooperación con } \\
\text { los alumnos, como fuente para la } \\
\text { generación de ideas en la actividad } \\
\text { innovadora. }\end{array}$ \\
\hline & $\begin{array}{l}\text { Disponibilidad y eficacia de sis- } \\
\text { temas formales o informales para } \\
\text { compartir recursos con los alumnos. }\end{array}$ \\
\hline
\end{tabular}




\begin{tabular}{|c|c|}
\hline $\begin{array}{l}\text { Relación con el } \\
\text { cliente para crear } \\
\text { conocimiento }\end{array}$ & $\begin{array}{l}\text { Disponibilidad y eficacia de los } \\
\text { sistemas de comunicación con los } \\
\text { alumnos }\end{array}$ \\
\hline \multirow{3}{*}{$\begin{array}{l}\text { Relación con el } \\
\text { proveedor para } \\
\text { crear conocimiento }\end{array}$} & $\begin{array}{l}\text { Habilidad para desarrollar acuer- } \\
\text { dos de cooperación que generen } \\
\text { ventajas competitivas mediante } \\
\text { externalización productiva. }\end{array}$ \\
\hline & $\begin{array}{l}\text { Importancia de la cooperación con } \\
\text { los proveedores como fuente para } \\
\text { la generación de ideas en la activi- } \\
\text { dad innovadora. }\end{array}$ \\
\hline & $\begin{array}{l}\text { Disponibilidad y eficacia de los } \\
\text { sistemas de comunicación con los } \\
\text { proveedores. }\end{array}$ \\
\hline \multirow{4}{*}{$\begin{array}{l}\text { Alianzas } \\
\text { estratégicas } \\
\text { establecidas por } \\
\text { la institución con } \\
\text { competidores, } \\
\text { clientes, gobierno, } \\
\text { proveedores, centros } \\
\text { tecnológicos, } \\
\text { universidades } \\
\text { u otras } \\
\text { organizaciones. }\end{array}$} & $\begin{array}{l}\text { Disponibilidad de acuerdos de } \\
\text { cooperación con otras organiza- } \\
\text { ciones. }\end{array}$ \\
\hline & $\begin{array}{l}\text { Habilidad de la institución para } \\
\text { gestionar alianzas estratégicas.. }\end{array}$ \\
\hline & $\begin{array}{l}\text { Importancia de la cooperación } \\
\text { con expertos y firmas consultoras } \\
\text { como fuente para la generación de } \\
\text { ideas innovadoras. }\end{array}$ \\
\hline & $\begin{array}{l}\text { Importancia de la cooperación con } \\
\text { universidades e institutos de inves- } \\
\text { tigación como fuente para la gene- } \\
\text { ración de ideas innovadoras. }\end{array}$ \\
\hline \multirow{3}{*}{$\begin{array}{l}\text { Capacidad de } \\
\text { conocimiento } \\
\text { mediante la } \\
\text { interacción con } \\
\text { otros agentes }\end{array}$} & $\begin{array}{l}\text { Importancia de los programas públi- } \\
\text { cos de apoyo a la innovación como } \\
\text { fuente de generación de ideas. }\end{array}$ \\
\hline & $\begin{array}{l}\text { Importancia de las ferias, exposi- } \\
\text { ciones y reuniones como fuente } \\
\text { para la generación de ideas inno- } \\
\text { vadoras. }\end{array}$ \\
\hline & $\begin{array}{l}\text { Habilidad de la institución para } \\
\text { establecer relaciones con asocia- } \\
\text { ciones de consumidores y otras } \\
\text { organizaciones sin ánimo de lucro. }\end{array}$ \\
\hline \multirow{4}{*}{$\begin{array}{l}\text { Recursos de } \\
\text { conocimiento } \\
\text { compartido merced } \\
\text { a la localización } \\
\text { de la empresa en } \\
\text { distritos industriales }\end{array}$} & $\begin{array}{l}\text { Conocimiento diferencial aportado } \\
\text { por proveedores, suministradores } \\
\text { y subcontratistas. }\end{array}$ \\
\hline & $\begin{array}{l}\text { Acumulación de conocimientos } \\
\text { científicos y tecnológicos por las } \\
\text { organizaciones del entorno, en } \\
\text { forma de patentes, investigaciones, } \\
\text { entre otros. }\end{array}$ \\
\hline & $\begin{array}{l}\text { Facilidad para la transmisión in- } \\
\text { formal de innovaciones y conoci- } \\
\text { mientos. }\end{array}$ \\
\hline & $\begin{array}{l}\text { Permeabilidad de la estructura so- } \\
\text { cial local. }\end{array}$ \\
\hline \multirow{3}{*}{$\begin{array}{l}\text { Eficacia de } \\
\text { los esfuerzos } \\
\text { de difusión de } \\
\text { conocimientos por } \\
\text { la institución para } \\
\text { ampliar su base de } \\
\text { alumnos. }\end{array}$} & $\begin{array}{l}\text { Esfuerzo de difusión de conoci- } \\
\text { mientos por los académicos y di- } \\
\text { rectivos de la institución en publi- } \\
\text { caciones. }\end{array}$ \\
\hline & $\begin{array}{l}\text { Grado de desarrollo de acciones } \\
\text { para la educación de los alumnos } \\
\text { (como campañas informativas, } \\
\text { manuales, entre otras.) }\end{array}$ \\
\hline & $\begin{array}{l}\text { Presencia pública de la institución } \\
\text { en acciones de reconocimiento } \\
\text { (como premios, distinciones) }\end{array}$ \\
\hline
\end{tabular}

Como ya se ha especificado en la metodología aplicada, se han desarrollado dos circulaciones que han consistido en la entrega de la encuesta a los participantes. Luego de haber aplicado nuevamente la metodología de aproximación de perfiles cualitativos, el resultado de la circulación definitiva arrojó las cualificaciones que mejor representan la opinión del grupo de estos participantes. Este resultado esta vez fue determinado por variable, información indispensable para poder distinguir las percepciones de cada una de ellas, para los próximos diez años de la Universidad de Atacama.

Por lo tanto, el resultado para cada variable del capital intelectual se detalla en la Tabla 5. En este caso el resultado final ha arrojado un valor síntesis que ayuda considerablemente a identificar ese escenario futuro.

Tabla 5. Resultado medición de capital intelectual por variable.

\begin{tabular}{|c|c|c|}
\hline $\begin{array}{c}\text { Variable capital } \\
\text { humano }\end{array}$ & $\begin{array}{c}\text { Variable capital } \\
\text { estructural }\end{array}$ & $\begin{array}{c}\text { Variable capital } \\
\text { relacional }\end{array}$ \\
\hline $\begin{array}{c}\text { Cualificación } \\
\text { sintetizada }\end{array}$ & $\begin{array}{c}\text { Cualificación } \\
\text { sintetizada }\end{array}$ & $\begin{array}{c}\text { Cualificación } \\
\text { sintetizada }\end{array}$ \\
\hline$(4)$ & $(4)$ & $(4)$ \\
\hline $\begin{array}{c}\text { Se mantendrá } \\
\text { en los mismos } \\
\text { niveles }\end{array}$ & $\begin{array}{c}\text { Se mantendrá } \\
\text { en los mismos } \\
\text { niveles }\end{array}$ & $\begin{array}{c}\text { Se mantendrá } \\
\text { en los mismos } \\
\text { niveles }\end{array}$ \\
\hline
\end{tabular}

Los escenarios planteados nos han permitido elaborar cuadros sintetizados, producto del ejercicio de pronóstico. Según las tablas de resultados del escenario exploratorio de la Universidad de Atacama, para fines del 2022 se puede deducir que $0,2 \%$ de los indicadores experimentarán un severo desmejoramiento, $8,8 \%$ de los indicadores experimentará desmejoramiento, $15 \%$ experimentará un leve desmejoramiento, $39 \%$ se mantendrán en los mismos niveles; $27 \%$ experimentará una leve mejora; $8,3 \%$ de los indicadores experimentará una mejoría clara y finalmente $1,7 \%$ experimentará un severo mejoramiento.

Por otro lado, los resultados arrojaron que las variables: capital intelectual humano, capital intelectual estructural y capital intelectual relacional no experimentarán mejoría pues se mantendrán en los mismos niveles.

Obsérvese que los resultados son expresados en forma linguística. La razón de ello es razonable, pues independiente de que todos los indicadores pueden 
ser gestionados en forma cuantitativa, sus valores por parte del grupo de expertos se debe expresar verbalmente principalmente por su imposibilidad de ser contabilizados. Lejos de obtener resultados numerológicos se obtienen expresiones lingüísticas que reflejan percepciones de una comunidad. Sabemos que se trata, por definición, del manejo de problemas no estructurados, llenos de incertidumbre y difíciles de cuantificar.

Consideramos que en materia de capital intelectual no arrojará al menos para finales del 2022 resultados halagadores, ya que se mantendrá en los mismos niveles. Esto debe llamar a la reflexión. Así se podrán tomar decisiones anticipadas, decisiones estratégicas que ayudarán a reducir la incertidumbre en el manejo de elementos intangibles, en una organización pública.

\section{CONCLUSIONES}

Los resultados que se han obtenido permiten concluir que la percepción de los funcionarios de la Universidad de Atacama en cuanto a las variables de capital intelectual podrían mantenerse en los mismos niveles. Esto, para los próximos diez años, ya que el valor síntesis que se calculó de acuerdo con la técnica de perfiles cualitativos arrojó una estabilización en el valor (4), valor que corresponde a la expresión definida en la escala Likert propuesta. Lo mismo ocurrió para las tres variables, es decir, para capital intelectual humano, capital intelectual estructural y capital intelectual relacional.

Es un resultado inesperado para los investigadores, porque se ha demostrado mediante la teoría la importancia del capital intelectual en el desarrollo de las organizaciones. Así, se puede deducir que el resultado obedece a la ausencia de líderes que incentiven el compromiso explícito, la asignación de recursos, una cultura acorde y política de recursos humanos en el desarrollo de ese capital intelectual.

Creemos que este estudio prospectivo contribuye a dejar en evidencia las variables intangibles que se deben mejorar, ya que estas están relacionadas con los procesos de acreditación en sus distintos niveles como son: la gestión institucional, la docencia de pregrado, la vinculación con el medio y la investigación.
Se debe considerar que la gran limitación de la investigación ha consistido en lo dificultoso que resulta medir elementos intangibles por su propia naturaleza. En este sentido, esta limitación ha privado a los investigadores a realizar una cuantificación exacta. No obstante ello, creemos que nuestro estudio ofrece un primer acercamiento.

\section{AGRADECIMIENTOS}

Este artículo es parte del Proyecto DIUDA-2011 de la Universidad de Atacama. En esta instancia, los autores agradecen a todos los que aportaron en la investigación.

\section{REFERENCIAS}

[1] L. Alcaniz, F. Gómez-Bezares and R. Roslender. "Theoretical perspectives on intellectual capital: A backward look and a proposal for going forward". Accounting Forum. Vol. 35, Issue 2, pp. 104-117. 2011.

[2] K. Sveiby. "The new Organizational Wealth: Managing and measuring knowledge based assets". Berret-Koehler Publishers. First Edition. Inc. San Francisco, California, Estados Unidos. 1997.

[3] L.A. Joia. "The impact of government to government endeavors on the intellectual capital of public organizations". Government Information Quarterly. Vol. 25, Issue 2, pp. 256-277. 2008.

[4] J. Guthrie, F. Ricceri and J. Dumay. "Reflections and projections: A decade of Intellectual Capital Accounting Research". The British Accounting Review. Vol. 44, Issue 2, pp. 68-82. 2012.

[5] M. Monagas-Docasal. "El Capital Intelectual y la Gestión del Conocimiento". Ing. Ind., Vol. 33 N² 2. La Habana, Cuba. 2012.

[6] M. Osorio. "El capital intelectual en la gestión del conocimiento". Editorial Ciencias Médicas. Vol. 11 N$^{\circ}$ 6. La Habana, Cuba. 2003.

[7] E. Rodríguez y A. Palma. "Desafíos de la Educación Superior en la economía del conocimiento". Ingeniare. Revista chilena de ingeniería. Vol. $18 \mathrm{~N}^{\circ}$ 1, pp. 8-14. 2010.

[8] M. León, G. Ponjuán y D. Torres. "Panorámica sobre la medición del conocimiento organizacional". Editorial Ciencias Médicas. Vol. 19 N ${ }^{\circ}$ 6. La Habana, Cuba. 2009. 
[9] S. Didou. "Internacionalización y proveedores externos de Educación Superior en los países de América Latina y en el Caribe: Principales problemáticas". Dpto. de Investigaciones Educativas/Centro de Investigación y de Estudios Avanzados-UNESCO. Ciudad de México, México. 2005.

[10] P. Molina, M. Arango y S. Botero. "Análisis del valor agregado del conocimiento. Caso aplicado en una Institución de Educación Superior". Revista Facultad de ciencias económicas: Investigación y reflexión. Universidad Militar Nueva Granada. Vol. XVIII No 2, pp. 95-105. Bogotá, Colombia. 2010.

[11] J. González y M. Rodríguez. "Modelos de Capital Intelectual y sus indicadores en la Universidad Pública". Cuadernos de Administración. № 43. Cali, Colombia. 2010.

[12] E. Rodríguez. "Gestión del conocimiento y eficacia de las organizaciones: Un estudio empírico en instituciones públicas". Interciencia. Vol. $32 \mathrm{~N}^{\circ} 12$, pp. 820-826. Caracas, Venezuela. 2007.

[13] Y. Marcano y R. Talavera. "Aproximación para el diseño de una red de conocimiento: Intra y Extra Universitaria". Revista de Ciencias Sociales. Vol. 15 N$^{\circ}$ 2, pp. 284-296. Maracaibo, Venezuela. 2009.

[14] J. Arapé. "Manual de metodologías. Tomo I: Marco Teórico-conceptual. Técnicas de escenarios, el pronóstico y la prospectiva en la formulación de políticas públicas". Programa de Prospectiva Tecnológica de la Secretaría de las Naciones Unidas para el Desarrollo Industrial. (ONUDI). Venezuela. 2000.

[15] J. Arapé. "Manual de metodologías. Tomo III: La Técnica Delphi”. Programa de Prospectiva Tecnológica de la Secretaría de las Naciones Unidas para el Desarrollo Industrial (ONUDI). Venezuela. 2000.

[16] M. Carreño. "El método Delphi: Cuando dos cabezas piensan más que una en el desarrollo de guías de práctica clínica". Revista Colombiana de psiquiatría. Vol. 38 No 1. Bogotá, Colombia. 2009.

[17] S. Sleimen, M. Rojas y M. Coringrato. "VIII Coloquio Internacional sobre Gestión Universitaria en América del Sur. Docencia, investigación y extensión universitaria desde la perspectiva del capital intelectual: Un aporte a la evaluación institucional". 2008.

[18] C. Devece, D. Palacios y F. Garrigós. "Un estudio Delphi para la medición del Capital Intelectual en la empresa". Departamento de Administración de empresa y Marketing. Universidad Jaume I de Castellón. Castellón de la Plana, España. 2002.

[19] J. Rodríguez, L. Aldana y N. Villalobos. "Método Delphi para la identificación de prioridades de Ciencia e Innovación Tecnológica". Revista Cubana de Medicina Militar. Vol. 39 Nº 3-4. 2010. 